\title{
Soft gamma-ray emission from the V404 Cygni outburst observed with INTEGRAL/PICsIT
}

\author{
Piotr Lubiński* \\ Institute of Physics, Zielona Góra University, Poland \\ E-mail: P.Lubinski@if.uz.zgora.pl
}

\begin{abstract}
Angela Bazzano, Lorenzo Natalucci, Pietro Ubertini
INAF, Istituto di Astrofisica e Planetologia Spaziali, Roma, Italy

E-mail: angela.bazzano@iaps.inaf.it, lorenzo.nataluccieiaps.inaf.it, pietro.ubertini@iaps.inaf.it
\end{abstract}

\section{Małgosia Sobolewska}

Harvard Smithsonian Center for Astrophysics, Cambridge, USA;

Nicolaus Copernicus Astronomical Center, Warsaw, Poland

E-mail: msobolewska@cfa.harvard.edu

\section{Piotr Życki}

Nicolaus Copernicus Astronomical Center, Warsaw, Poland

E-mail: ptz@camk.edu.pl

\begin{abstract}
V404 Cygni during its 2015 outburst was the brightest source ever observed by INTEGRAL in the soft gamma-ray band. This made possible to detect the source with the IBIS high energy detector PICsIT in many single pointings $(\approx 1 \mathrm{~h}$ long) allowing to study the evolution of the gamma-ray emission over the whole event period. In general, the PICsIT flux in the $255-340 \mathrm{keV}$ band correlates with the hard X-ray flux measured by INTEGRAL/ISGRI, although we observe a saturation of the gamma-ray emission from the source. The spectra extracted for several short periods of the outburst were modelled with a Comptonization model. The plasma temperature remains stable despite a variation of the Compton parameter. We found a strong Compton reflection and a hint for a non-thermal emission occurring during some phases of the outburst. Our preliminary analysis do not show a presence of the positron annihilation line in the V404 Cygni spectra from the 2015 outburst.
\end{abstract}

11th INTEGRAL Conference Gamma-Ray Astrophysics in Multi-Wavelength Perspective 10-14 October 2016

Amsterdam, The Netherlands

\footnotetext{
* Speaker.
} 


\section{Introduction}

The 2015 outburst of V404 Cygni triggered dozens of studies over the entire electromagnetic emission range. Many of those studies were based on the INTEGRAL's [13] observations carried out almost continuously for about a month. Thanks to the enormous brightness of the source it was possible to explore its spectral properties in the hard X-ray and soft $\gamma$-ray bands to time scales down to minutes $[6,7,9]$.

Spectral analysis of the initial phase of the outburst was performed with the INTEGRAL's ISGRI and SPI detectors spectra [6]. The fit with a Comptonization model applied to 6 spectra sampled over one of the flares resulted in a relatively low electron plasma temperature $k T_{e} \approx 40$ $\mathrm{keV}$. Only for one of the spectra an addition of the reflection component improved the fit. Another set of sample spectra from the same period was analysed with the two continuum components model, including a low-temperature Comptonization and an exponentially cut-off power-law at high energy [7]. Fitted values of $k T_{e}$ were within $21-53 \mathrm{keV}$ range, whereas the high-energy cutoff of the second component was found within 200-300 keV range.

Recently a set of 602 ISGRI spectra extracted for the whole INTEGRAL observing period was analysed with two Comptonization models [9]. Fitted $k T_{e}$ values formed a relatively narrow distribution centered around $40 \mathrm{keV}$, whereas the plasma optical depth values were found in a broad range between 0 and 6 . The authors identified two main branches in the fit results: a hard one with $k T_{e}$ anticorrelated with the hard X-ray flux and a soft flaring one, with a reversed correlation.

One of the most intriguing INTEGRAL results, not only in the V404 Cygni outburst context, is a varying positron annihilation component found in the SPI spectra [11]. This result, if confirmed, will have important consequences for both the microquasar physics and the models of positron production in Milky Way.

In this paper we present for the first time the results of the V404 Cygni observations made with PICsIT, INTEGRAL's $\gamma$-ray detector rarely used due to an exclusively high energy band and a demanding data analysis. Besides a preliminary analysis of the PICsIT light curves and several 1-hour spectra we compare our summed PICsIT spectra with the SPI spectra, in which the annihilation features were found.

\section{INTEGRAL/PICsIT detector}

The PICsIT detector [3] is the lower detector layer of the coded-mask IBIS telescope [12] of the INTEGRAL satellite. The detection plane is an array of 64x64 (4096) CsI pixels tuned to detect $\gamma$ rays in the $170 \mathrm{keV}-8 \mathrm{MeV}$ nominal energy band. The soft $\gamma$-ray band is very strongly affected by the cosmic-ray induced instrumental background and data analysis is quite demanding. The standard OSA software [1], based on an image deconvolution, allows for a detection of only a couple of brightest sources [2]. In addition, due to the limited telemetry band PICsIT is routinely not operating in the event-by-event mode. This makes impossible to filter out so-called track events, affecting especially the lowest energy range of PICsIT, below $250 \mathrm{keV} \mathrm{[10].}$

A mean number of the source photons registered by a single PICsIT pixel during a single pointing (science window, 0.5-2 h) is of order of 1 for the strongest persistent sources (Crab, Cyg $\mathrm{X}-1$ ). For such a small number of counts an approximation of the Poisson distribution with a normal 


\begin{tabular}{lccc}
\hline & SPI & PICsIT & SPI/PICsIT \\
\hline Area & $500 \mathrm{~cm}^{2}$ & $2900 \mathrm{~cm}^{2}$ & 0.2 \\
Volume & $3500 \mathrm{~cm}^{3}$ & $8800 \mathrm{~cm}^{3}$ & 0.4 \\
Number of pixels & 19 & 4096 & 0.005 \\
Veto background reduction & $4 \%$ & $40 \%$ & 10 \\
Photon attenuation $\left(\propto Z^{3}\right)$ & $\mathrm{Ge}(\mathrm{Z}=32)$ & $\mathrm{CsI}(\mathrm{Z}=55 / 53)$ & 0.2 \\
\hline
\end{tabular}

Table 1: Comparison of the SPI and PICsIT detectors.

distribution definitely does not work. Therefore, an alternative software for the PICsIT detector was developed [4]. A main ingredient of the new method is a direct handling of the probability density functions (PDFs) for both background and source. Instead of an image deconvolution the source spectra are extracted directly using the pixel illuminationn function (PIF). The PIFs for each source are computed through a ray-tracing, enabling a better modelling of the instrument than the mask model included in the standard OSA software. The third element of the new software is a careful modelling of the instrumental background, based on studies of the background variability over a single satellite revolution period. The PICSIT spectral extraction software allowed for a detection of 12 sources listed in [4]. Until now only the results for Cyg X-1 were used for a scientific analysis $[5,14]$, however, the tests show that the number of detected sources should exceed 20.

The method described in [4] assumes that there is only one detectable source in the field of view of the instrument. This is a good approximation because the detectable celectial objects are usually well separated. In the case of the V404 Cygni analysis a proximity of Cyg X-1 (about 5.5 degrees) violates this assumption. This delayed our analysis because a new version of the software had to be developed and tested, to deal with three PDFs of two sources and background at once. In addition, we had to test if the background modelling neglecting the source emission will be still valid for the V404 Cygni emission reaching at maximum about $10 \%$ of the background rate.

Since in the Sec. 4 we compare the SPI and PICsIT spectral results, we briefly discuss here on the sensitivity of these two instruments. In Table 1 we collected basic parameters of both instruments relevant for the continuum sensitivity. Both detector area and volume are much larger for PICsIT. In addition, due to the heavier elements used as a detecting material PICsIT has about 5 times larger electronic stopping power for high-energy photons than SPI. On the other hand, the anti-Compton veto system is much more efficient in case of SPI, providing better background reduction. Moreover, the heavier material of the PICsIT detector means much higher level of induced instrumental background. Another important factor affecting the sensitivity of the codedmask instruments is the number of pixels. Large number of smaller PICsIT pixels allows for a better determination of the mask shadow over the detector plane. This in turn enables a better disentanglement of the source emission from the background radiation.

Surely for the background-dominated soft $\gamma$-ray band the most important factor determining the detector sensitivity is the background level. Therefore, taking into account the parameters presented in Table 1 one can expect that the continuum sensitivity of SPI and PICsIT should be more or less similar. Figure 1 confirms this presumption. The three sensitivity curves shown there were taken from the IBIS and SPI Observer manuals [15, 16]. IBIS and SPI perfomance cannot be directly compared due to the different observation times assumed. In addition, in case of 
PICsIT the sensitivity estimate based on the standard imaging software is rather uncertain. For this reason we present more reliable results based on the studies of the actual noise level of the PICsIT detector, determined for a large number of empty-field observations with the Poisson PDF method applied [4]. In estimating the sensitivity, detection levels are converted to flux using actual Crab observations. As can be seen in Fig. 1, indeed the sensitivity of SPI and PICsIT in the 250-2000 $\mathrm{keV}$ band is quite similar.

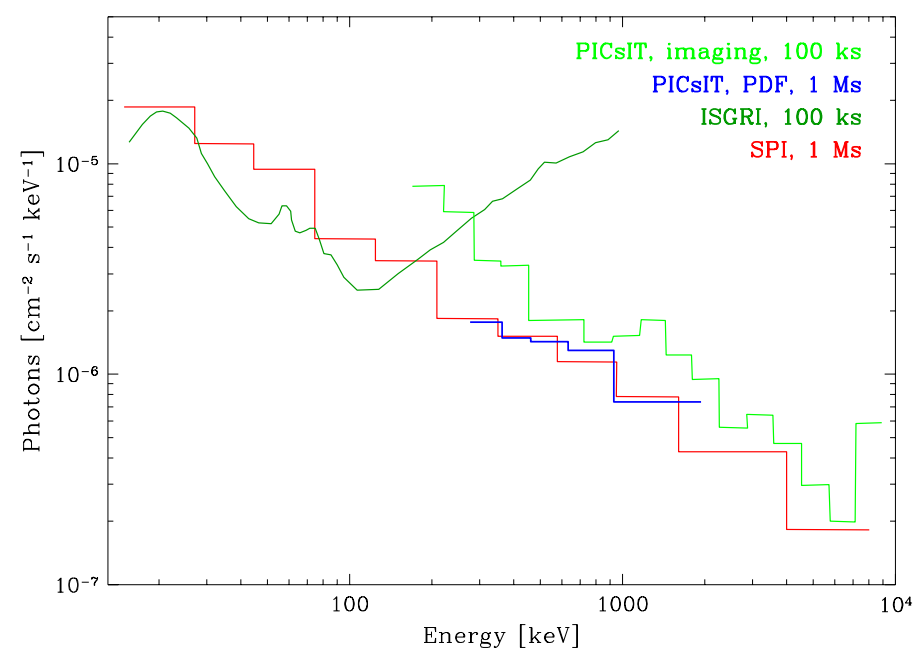

Figure 1: Continuum sensitivity of the INTEGRAL gamma-ray detectors. ISGRI and PICsIT imaging data were taken from [15], SPI data were taken from [16]. PICsIT estimates based on the PDF method were taken from [4].

\section{Light curves}

The outburst of V404 Cygni provided us the best ever PICsIT data for hour-scale variation study. The source emission in the $255-340 \mathrm{keV}$ band reached at maximum about 24 counts per second (cps). This corresponds to almost 6 Crab units (the mean Crab count rate for this band is around $4.4 \mathrm{cps}$ ). In Fig. 2 we present the V404 Cygni light curves in three energy bands, obtained with the ISGRI and PICsIT detectors. In general, the PICsIT light curve clearly follows both ISGRI light curves, especially the higher energy one (100-200 keV). A chaotic variability of the source characterizes the first five days of the outburst, till MJD 57197.5. Then, the light curves for the rest of revolution 1556 and a major part of revolution 1557 become much more smooth. This allows us to observe clearly several episodes of breaking correlation between the emission below $40 \mathrm{keV}$ and above $100 \mathrm{keV}$, as seen around MJD 57198 and during the two highest-rate periods in revolution 1557.

Correlations between the $255-340 \mathrm{keV}$ emission and the emission in $22-40 \mathrm{keV}$ and 100 $200 \mathrm{keV}$ are shown in Fig. 3. For the softer ISGRI band $(22-40 \mathrm{keV})$ there is only some overall correlation. Below $1000 \mathrm{cps}$ measured for hard X-rays, the PICsIT soft $\gamma$-ray count rate exhibits a large range of values, corresponding either to a lack of or marginal detection $(<5 \mathrm{cps})$, or to a clear detection $(>5 \mathrm{cps}$ ). The highest $255-340 \mathrm{keV}$ emission is observed for ISGRI count rate around 
$1500 \mathrm{cps}$, much below its highest level ( $>5000 \mathrm{cps}$ ). For the highest hard X-ray emission the $\gamma$-ray emission appears to saturate at the level around $10 \mathrm{cps}$.

On the other hand, correlation between both soft $\gamma$-ray bands, 100-200 keV and 255-340 keV, is much stronger. Below $\approx 75 \mathrm{cps}$ in the $100-200 \mathrm{keV}$ band the source is rarely detected in the 255-340 keV band. For higher count rates observed with ISGRI ( $>75 \mathrm{cps}$ ) V404 Cygni is very bright in the $255-340 \mathrm{keV}$ band. However, we observe again a saturation of the PICsIT count rates within the 5-15 cps range. The only exception from this trend are three points corresponding to the beginning of revolution 1557, when the source emission was extremely hard.

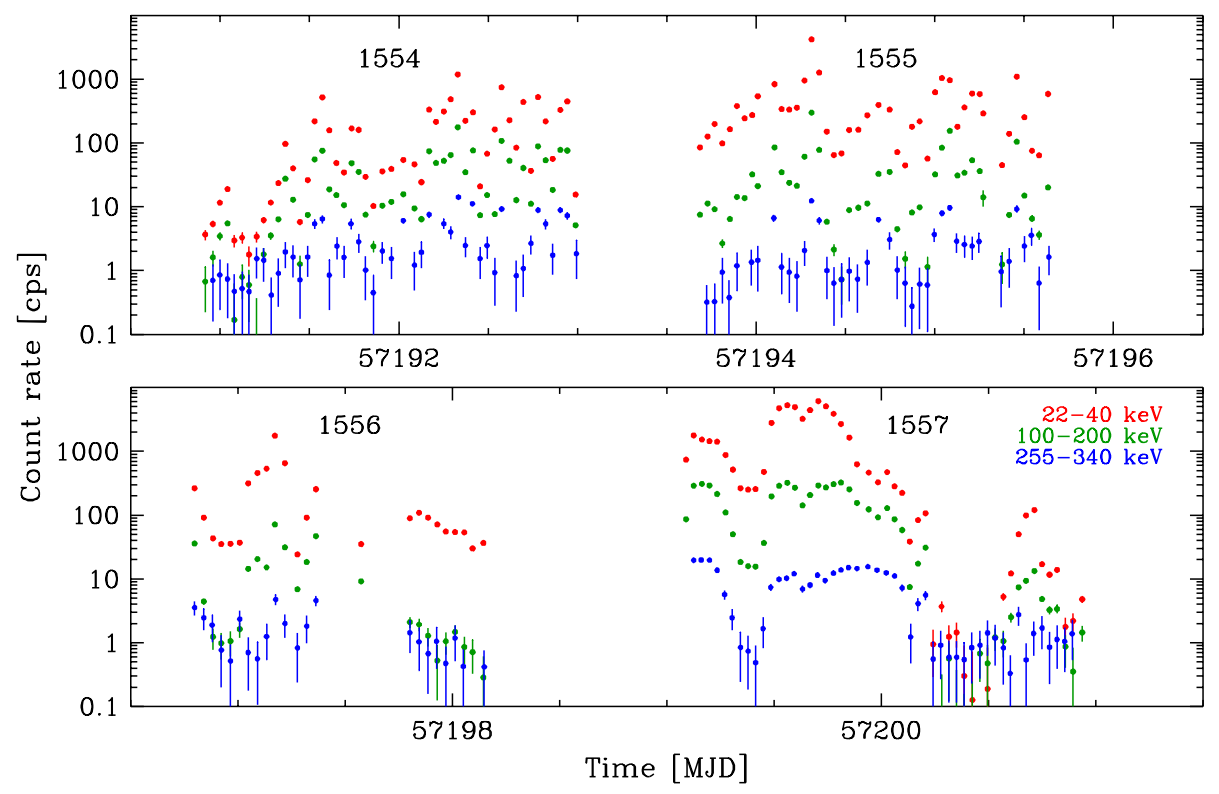

Figure 2: Light curves of V404 Cygni outburst, period 2015-06-17 22:30 - 2015-06-27 21:53 (INTEGRAL revolutions 1554-1557). Energy bands: 22-40 keV (ISGRI, red), 100-200 keV (ISGRI, green), 255-340 $\mathrm{keV}$ (PICsIT, blue).
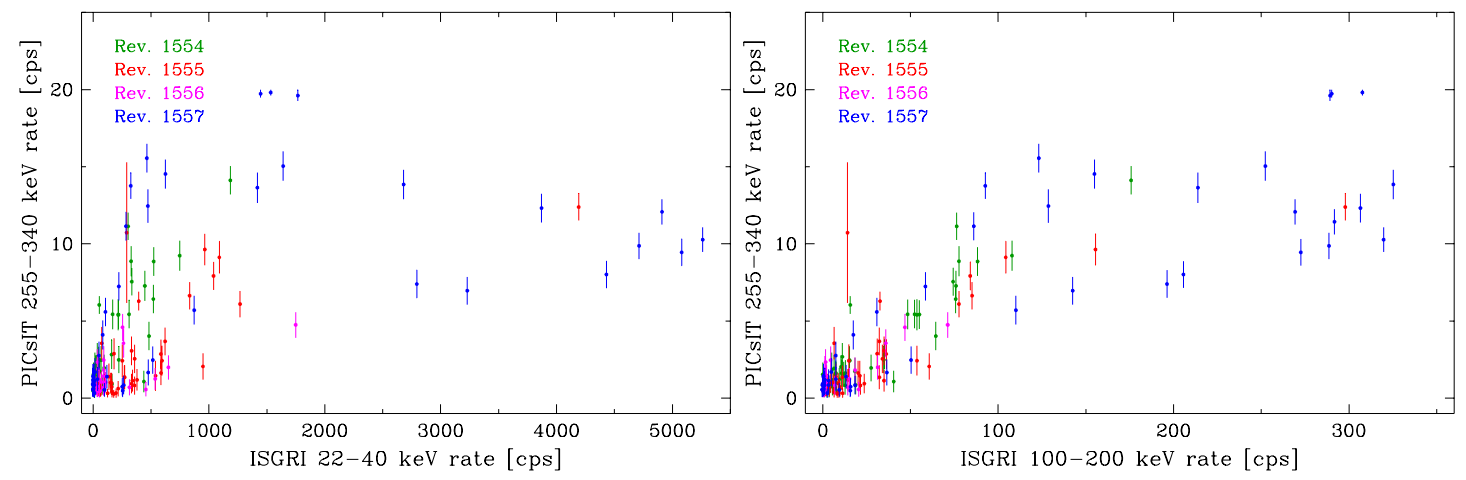

Figure 3: Correlations between the PICsIT count rate in the $255-340 \mathrm{keV}$ band and the ISGRI count rates in the 22-40 keV (left) and 100-200 keV (right) bands.

The enormous $\gamma$-ray brightness of the V404 Cygni outburst justifies light curve extraction for even higher energies. Figure 4 presents the PICsIT light curve computed for the 532-930 keV 
band. We can state a 3- $\sigma$ detections only for two science windows, around MJD 57195.1 and MJD 57199.7. However, there are several more points seen in the revolution 1557 data with a relatively high flux in that band. They are correlated with the high ISGRI and PICsIT count rate in the softer $\gamma$-ray bands. We plan to explore the $\gamma$-ray variability of V404 Cygni on so short time scales in a forthcoming paper, after an optimisation of the signal-to-noise ratio with a better tuning of the energy channels and a more sophisticated background modelling.

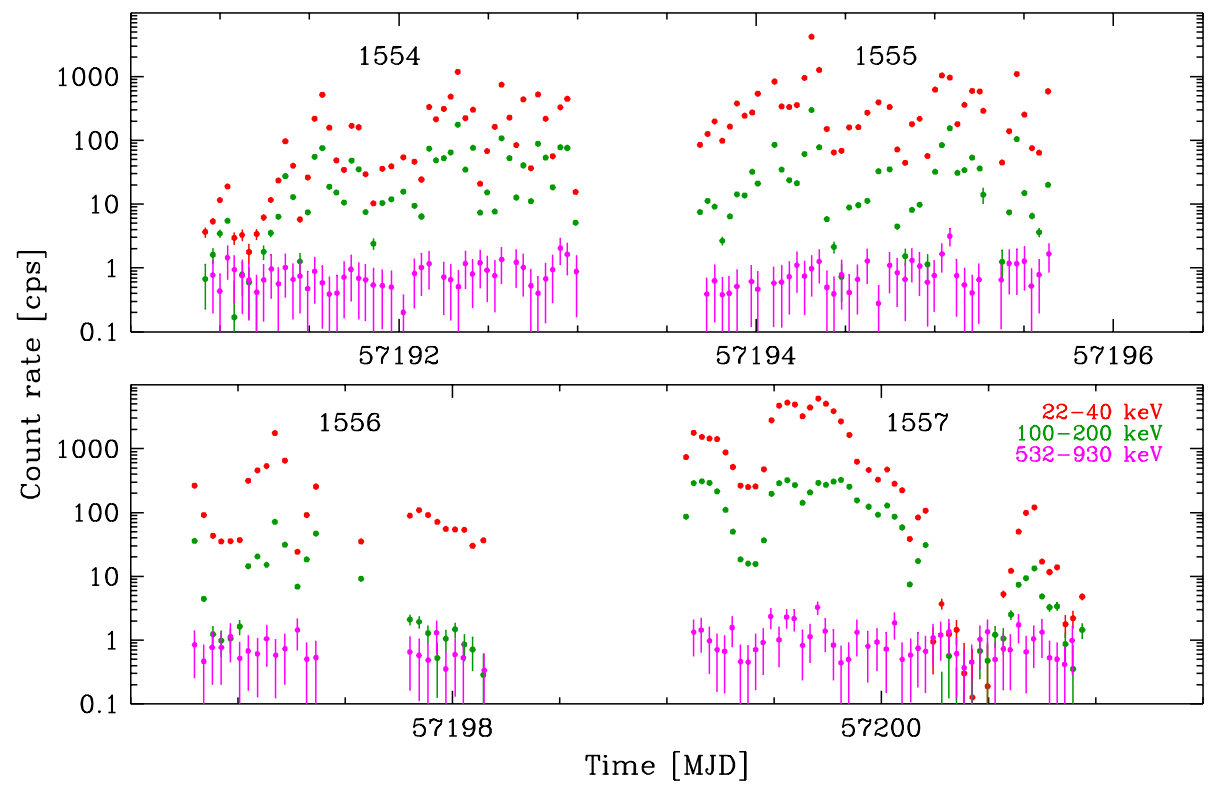

Figure 4: Light curves of V404 Cygni outburst. Energy bands: 22-40 keV (ISGRI, red), 100-200 keV (ISGRI, green), 532-930 keV (PICsIT, purple).

\section{Spectral results}

PICsIT on-board histograms are summed over entire science window. Thus, this is a minimal time period for the PICsIT spectral analysis. We have selected several science windows with a strong $\gamma$-ray emission to test the Comptonization model applied to the short-time spectra. The PICsIT spectra were fitted together with the ISGRI and JEM-X spectra. The latter were added to constrain an absorption component. The results of the spectral fitting are presented in Table 2. For fitting we used XSPEC v. 12.9.0n. The main continuum component is a Comptonization spectrum that we modelled by COMPPS, folded with a simple absorption model (WABS). We allowed for a free normalization of each spectrum (fixed at 1 for ISGRI, fitted $c_{J}$ for JEM-X and $c_{P}$ for PICsIT). The best fitted plasma temperature, $k T_{e}$, are in a narrow range between 60 and $80 \mathrm{keV}$. All values of the Compton parameter $y$ are well below 1, pointing to a rather weak Comptonization regime. For all three analysed spectral sets we found a moderate absorption $\left(N_{H}\right)$ and a quite strong reflection. The reflection strength parameter $R$ is larger than 1, i.e. the value expected for a source illuminating an infinite slab. Large $R$ value can be partly explained by a convex shape of the accretion disc surrounding the primary photon source. 
As mentioned earlier, spectral analysis of the V404 Cygni data must be preceded by a careful selection of periods over which the spectra are integrated. Figure 2 and the $y$ results in Table 2 show a rapid spectral variability, with the spectral slope changing within hours. Therefore, an interpretation of the models fitted to the spectra summed over an entire revolution can be misleading if the mean spectral shape does not correspond to any of the true spectral shapes through which the source evolves. In addition, V404 Cygni emission frequently declines to the non-detectable level and summing up data with those dim periods included considerably decreases the signal-tonoise ratio. Nevertheless, in Figs. 5 and 6 we present the spectra summed for longer periods. This is done for a purpose of comparison with the SPI spectra presented in [11], with the strong annihilation features reported.

The ISGRI and PICsIT spectra summed for entire revolutions 1555 and 1557 showed a much lower flux that those of SPI. Thus, for comparison we have excluded data corresponding to the dim state of the object within these two revolutions. With this procedure we obtained an overall agreement between the SPI and PICsIT fluxes in the 300-500 keV band. In Figures 5 and 6 we show also the 3- $\sigma$ noise level estimate for PICsIT observation integrated over similar exposure time [4]. In case of revolution 1555 the V404 Cygni emission is detected up to about $500 \mathrm{keV}$. No broad annihilation line is detected and the data above $500 \mathrm{keV}$ are compatible with the background fluctuations. On the other hand we observe a discrepancy between the ISGRI and PICsIT data at around $300 \mathrm{keV}$. This is presumably an effect of a rapid source variability during this revolution, with count rates varying over several orders of magnitude.

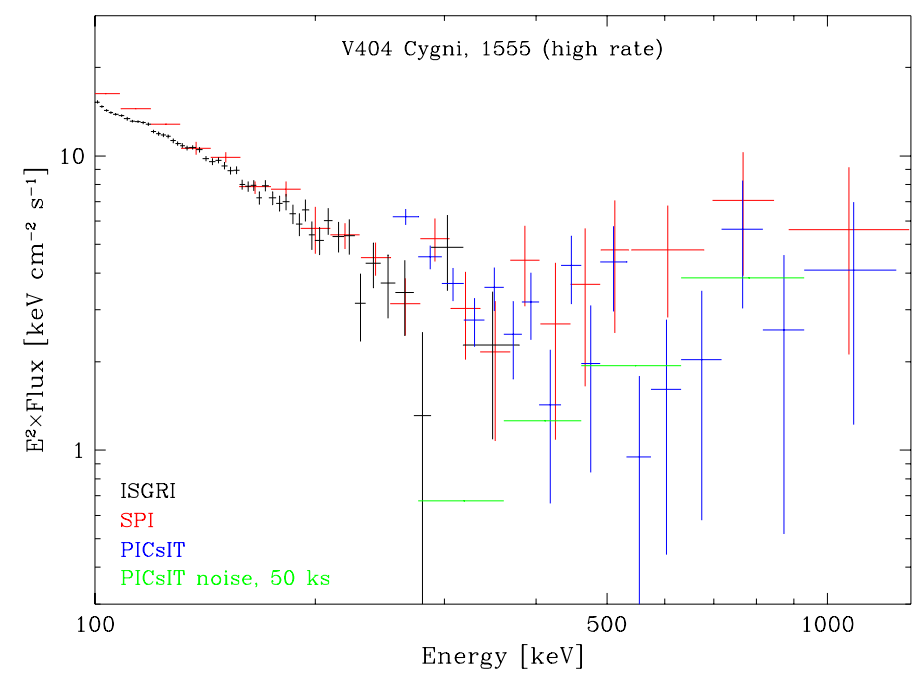

Figure 5: Summed ISGRI (black) and PICsIT (blue) spectra corresponding to bright phase of the V404 Cygni outburst during revolution 1555. The SPI spectrum (red) of entire revolution 1555 was read from fig. 1 of [11]. PICsIT noise level (3- $\sigma$ detection limit) is shown with green lines.

The PICsIT spectrum shown in Fig. 6 is relatively smooth and the narrow annihilation feature found in the SPI spectrum at around $470 \mathrm{keV}$ is not seen. We observe a deviation in the ISGRI spectrum at around $300 \mathrm{keV}$ but its statistical meaning is weak. Interestingly, the PICsIT spectrum is much flatter than that of revolution 1555 and the object emission is marginally detected up to about $600 \mathrm{keV}$. This confirms our observation based on the 532-930 keV light curve shown in 


\begin{tabular}{cccccccc}
\hline Science window & $\begin{array}{c}k T_{e} \\
{[\mathrm{keV}]}\end{array}$ & $R$ & $y$ & $\begin{array}{c}N_{H} \\
{\left[10^{22} \mathrm{~cm}^{-2}\right]}\end{array}$ & $c_{P}$ & $c_{J}$ & $\chi^{2} /$ d.o.f. \\
\hline 155500190010 & $69_{-8}^{+9}$ & $2.8_{-0.4}^{+0.6}$ & $0.68_{-0.04}^{+0.04}$ & $2.2_{-1.0}^{+1.0}$ & $0.85_{-0.13}^{+0.15}$ & $1.06_{-0.07}^{+0.07}$ & $58.1 / 155$ \\
155700140010 & $63_{-8}^{+8}$ & $3.0_{-0.8}^{+0.8}$ & $0.65_{-0.04}^{+0.03}$ & $2.5_{-1.1}^{+1.1}$ & $0.86_{-0.12}^{+0.14}$ & $0.98_{-0.08}^{+0.08}$ & $58.2 / 143$ \\
155700190010 & $80_{-9}^{+10}$ & $2.5_{-0.5}^{+0.5}$ & $0.43_{-0.04}^{+0.04}$ & $4.7_{-1.3}^{+1.2}$ & $0.87_{-0.13}^{+0.14}$ & $0.91_{-0.07}^{+0.07}$ & $79.9 / 142$ \\
\hline
\end{tabular}

Table 2: Results of the Comptonization model fit to the joint JEM-X, ISGRI and PICsIT spectra of V404 Cygni collected during several science windows. Fitted parameter symbols are explained in the text.

Fig. 3, that the $\gamma$-ray spectra were very hard during some periods of this revolution. In future we will analyse this emission with a careful selection of the integration periods and an optimal energy binning.

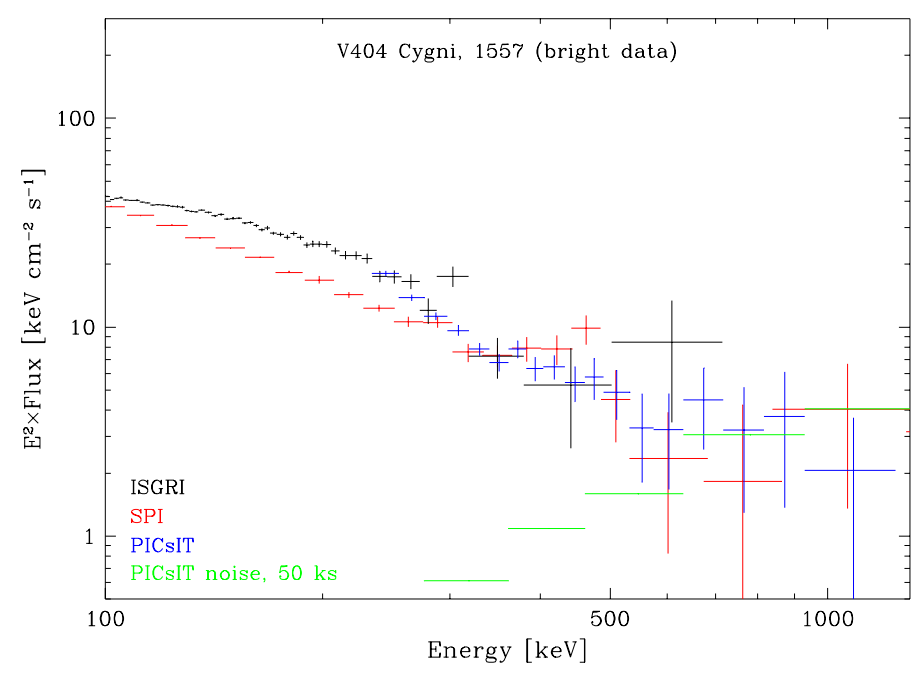

Figure 6: Summed ISGRI (black) and PICsIT (blue) spectra corresponding to bright phase of the V404 Cygni outburst during revolution 1557. The SPI spectrum (red) of entire revolution 1557 was read from fig. 1 of [11]. PICsIT noise level (3- $\sigma$ detection limit) is shown with green lines.

\section{Summary}

The 2015 outburst of V404 Cygni brought a wealth of data on the most violent mode of an accretion in black hole systems thanks to a large source luminosity that triggered numerous observations in various energy bands. INTEGRAL's monitoring of the source lasted almost a month. Whereas the ISGRI and SPI analysis of the event was already reported by several groups, the PICsIT data remained unexplored. An exceptional brightness of V404 Cygni allowed us to study the variability and spectral properties of the $250-930 \mathrm{keV}$ emission registered by PICsIT on time scale of hours.

PICsIT count rate in the $255-340 \mathrm{keV}$ band is, in general, clearly correlated with the ISGRI count rate in the 100-200 keV band, despite the rapid and huge changes of the latter. On the contrary, a correlation of the $\gamma$-ray emission with the hard X-ray emission in the $22-40 \mathrm{keV}$ band of ISGRI is much weaker. To some extent this weakening of correlation is observed due to the 
saturation of the high-energy emission. Such a saturation of the $255-340 \mathrm{keV}$ signal within a range of 2-4 Crab units is observed over entire studied period, except for several hours around MJD 57199.15, when the V404 Cygni emission reached a level of 6 Crab units.

The ISGRI and PICsIT light curves of V404 Cygni extracted for different energy bands demonstrate fast changes of the spectral slope. We observe periods when for the same level of the hard $\mathrm{X}$-ray count rate the soft $\gamma$-ray count rates either remain high or drop to a non-detectable level. Close to the end of the outburst the source behaviour was less erratic and the $\gamma$-ray emission becomes very hard. The 532-930 keV light curve with several marginal detections of the V404 Cygni signal suggests a strong non-thermal emission, for the first time observed within such a short period.

Our preliminary spectral analysis of the V404 Cygni emission was applied to only three individual science windows data. We analysed joint JEM-X, ISGRI and PICsIT spectra with the model including a thermal Comptonization continuum and its reflection, both absorbed. This model gave an acceptable fit, thus at least for these three periods there is no need to include an additional, non-thermal component. We found the plasma temperature close to about $70 \mathrm{keV}$, i.e. a typical value for the accreting black hole systems. The Compton parameter values well below 1 suggest a relatively weak Comptonization, presumably due to a dilute plasma region. A strong Compton reflection component was found, possibly related to either the strong gravity effects or a large solid angle covered by a reflecting medium. If the reflecting medium is also a main absorber of the primary emission, it cannot block out too much that emission at high flux levels because we found relatively weak absorption for analysed spectra.

We have preliminarily compared SPI spectra with those obtained by PICsIT for similar periods without a significant detection of the annihilation features. Notwithstanding, we observe with PICsIT a high-energy tail in one of the summed spectra, compatible with the corresponding SPI spectrum. In future we will continue this analysis of the spectra with a various data selection criteria, including the spectral integration time and different width of the energy channels.

\section{Acknowledgments}

P.L. was supported by the Polish National Science Centre grant UMO-2014/13/B/ST9/00570. Italian co-authors acknowledge support from the ASI/INAF agreement 2013.025.R1.

\section{References}

[1] T.J.-L. Courvoisier et al., The INTEGRAL Science Data Centre (ISDC), A\&A 411 (2003) 53.

[2] L. Foschini et al., IBIS/PICsIT Instrument Specific Software Scientific Validation Report, 2007, http://www.isdc.unige.ch/integral/download/osa/doc/10.2/osa_sci_val_picsit.pdf.

[3] C. Labanti et al., The Ibis-Picsit detector onboard Integral, A\&A, 411 (2003) 149.

[4] P. Lubiński, Analysis of extremely low signal-to-noise ratio data from INTEGRAL/PICsIT, A\&A 496 (2009) 557.

[5] J. Malzac et al., An intense state of hard X-ray emission of Cyg X-1 observed by INTEGRAL coincident with TeV measurements, A\&A 492 (2008) 527. 
[6] L. Natalucci et al., High Energy Spectral Evolution of V404 Cygni during the 2015 June Outburst as Observed by INTEGRAL, ApJ 813 (2015) 21.

[7] J.P. Roques et al., First INTEGRAL Observations of V404 Cygni during the 2015 Outburst: Spectral Behavior in the 20-650 keV Energy Range, ApJ 813 (2015) 22.

[8] J.P. Roques, E. Jourdain, High Energy Emission of V404 Cygni during 2015 outburst with INTEGRAL/SPI: Spectral analysis issues and solutions, 2016, eprint arXiv:1601.05289.

[9] C. Sanchez-Fernandez, J.J.E. Kajava, S.E. Motta, E. Kuulkers, Hard X-ray variability of V404 Cyg during the 2015 outburst, 2016, eprint arXiv:1608.08802.

[10] A. Segreto et al., Cosmic rays tracks on the PICsIT detector, A\&A, 411 (2003) 215.

[11] T. Siegert et al., Positron annihilation signatures associated with the outburst of the microquasar V404 Cygni, Nature 531 (2016) 341.

[12] P. Ubertini et al., IBIS: The Imager on-board INTEGRAL, A\&A 411 (2003) 131.

[13] C. Winkler et al., The INTEGRAL mission, A\&A, 411 (2003) 1.

[14] A.A. Zdziarski, P. Lubiński, M. Sikora, The MeV spectral tail in Cyg X-1 and optically thin emission of jets, MNRAS 423 (2012) 663.

[15] INTEGRAL 14th Announcement of Opportunity, IBIS Observer's Manual, 2016, http://integral.esac.esa.int/AO14/IBIS_ObsMan.pdf.

[16] INTEGRAL 14th Announcement of Opportunity, SPI Observer's Manual, 2016, http://integral.esac.esa.int/AO14/SPI_ObsMan.pdf. 